\title{
Fibrinogen Mediates Platelet-Polymorphonuclear Leukocyte Cooperation during Immune-Complex Glomerulonephritis in Rats
}

\author{
Xiaobo Wu, Miep H. Helfrich, ${ }^{*}$ Michael A. Horton, ${ }^{\ddagger}$ Larry P. Feigen, ${ }^{5}$ and James B. Lefkowith \\ Departments of Medicine and Molecular Biology and Pharmacology, Washington University School of Medicine, St. Louis, Missouri \\ 63110; *Department of Medicine and Therapeutics, University of Aberdeen, Aberdeen AB9 2Z0, Scotland; ${ }^{\ddagger}$ Imperial Cancer Research \\ Fund Haemopoiesis Research Group, Department of Haematology, St. Bartholomew's Hospital, London EC1A 7BE, United Kingdom; \\ and ${ }^{8}$ Searle Pharmaceuticals, Skokie, Illinois 60077
}

\begin{abstract}
The metabolic and functional alterations which occur during the acute phase of nephrotoxic nephritis (NTN) in rats, a model of immune-mediated glomerulonephritis, result from a cooperative interaction between PMNs and platelets (PLTs). In consequence, we hypothesized that fibrinogen (Fg) might play a critical role in this process and, accordingly, we found that defibrination of animals decreased both the acute phase proteinuria in NTN $(\sim 70 \%)$ as well as the influx of PLTs and PMNs into the glomerulus $(\sim 40-50 \%)$. In contrast, blockade of the PLT Fg receptor, $\alpha_{\text {IIb }} \beta_{3}$, with the RGD peptidomimetic SC-49992 decreased proteinuria ( $\sim 90 \%)$ without substantially altering the influx of PMNs or PLTs. Immunocytochemistry showed a marked increase in $\beta_{3}$ integrin expression in inflamed glomeruli which was prevented either by PMN or PLT depletion before disease induction. FACS $\otimes$ and immunocytochemical analysis of glomerular cell dissociates demonstrated that $\beta_{3}$ integrin expression was predominantly on intraglomerular PLTs. In vitro, activated PLTs stimulated the PMN respiratory burst, an interaction which could be inhibited by $\mathrm{Fg}$ receptor blockade. In sum, acute NTN is accompanied by a marked increase in glomerular $\beta_{3}$ integrin expression predominantly due to the influx of PLTs which localize to the glomerulus in a PMN-dependent fashion. Fg appears to serve a major role as a coactivating stimulus for PLT-PMNs in situ via $\alpha_{\mathrm{Ib}} \beta_{3}$, potentially mediating the PMN respiratory burst which contributes to proteinuria. Fg may also play a subsidiary role in PMN/PLT comigration. (J. Clin. Invest. 1994. 94:928-936.) Key words: glomerulonephritis • fibrinogen • $\beta_{3}$ integrin $\cdot$ neutrophil $\bullet$ platelet
\end{abstract}

\section{Introduction}

Nephrotoxic nephritis (NTN) ${ }^{1}$ in rats serves as a model system for immune-mediated glomerulonephritis in man $(1,2)$. Studies

Address correspondence to James B. Lefkowith, M.D., Box 8045, Division of Rheumatology, Washington University School of Medicine, St. Louis, MO 63110.

Received for publication 14 January 1994 and in revised form 6 May 1994.

1. Abbreviations used in this paper: $\mathrm{CD} 45$, leukocyte common antigen; $\mathrm{Fg}$, fibrinogen; HRP, horseradish peroxidase; NTN, nephrotoxic nephritis; PLT, platelet.

J. Clin. Invest.

(C) The American Society for Clinical Investigation, Inc. 0021-9738/94/09/0928/09\$2.00

Volume 94, September 1994, 928-936 into the pathogenesis of this disease model show that leukocytes are critical effectors of the glomerular damage and dysfunction in this disease model and the accompanying alterations in glomerular biochemistry $(1,2)$. Because of the central role played by leukocytes in this form of experimental glomerulonephritis, the mechanisms by which these cells enter glomeruli in the context of inflammation, become activated, and induce glomerular dysfunction are of topical concern.

With respect to PMNs (which mediate the acute phase of NTN) it has become clear that their emigration into glomeruli is a complex function of the activation of complement, the production of local cytokines, the upregulation of vascular adhesion molecules, and the expression of $\beta_{2}$ integrins on the PMNs (3-5). In addition, in recent work by our laboratory, it appears that platelets (PLTs), as well, are essential for the efficient influx of PMNs into the glomerulus and contribute to the acute glomerular injury (6). Specifically, we have found that PLTs are essential for the influx of PMNs into the glomerulus, the upregulation in glomerular leukotriene $\mathbf{B}_{4}$ production, and the evolution of proteinuria (6). Conversely, PMNs are essential for the influx of PLTs into the glomerulus as well as the upregulation in glomerular thromboxane production (6) and are clearly a major effector for producing proteinuria (7).

PLT-PMN interactions appear to be a common part of the acute inflammatory response and may be mediated via a myriad of different molecular mechanisms $(8,9)$. In an effort to broach this general issue in the context of NTN, we focused specifically on fibrinogen $(\mathrm{Fg})$. We hypothesized that $\mathrm{Fg}$ would play a critical role in the interaction of PLTs and PMNs in inflamed glomeruli based on prior in vivo data suggesting that defibrination/ fibrinolysis could ameliorate experimental glomerulonephritis (although not invariably) $(10,11)$ and on in vitro evidence that Fg could serve to activate PLTs and PMNs as well as play a role in their migration via effects on adherence and chemotaxis $(12,13)$. Underscoring the potentially important role of $\mathrm{Fg}$ in inflammation are data which show that $\mathrm{Fg}$ alone (even in the absence of complement or immunoglobulin) is sufficient to render inert materials proinflammatory (14).

By comparing and contrasting defibrination with $\mathrm{Fg}$ receptor blockade in vivo, by probing for $\beta_{3}$ integrin expression in vivo using immunocytochemical techniques, and by examining PLT-PMN interactions in vitro, we were able to establish a central role for $\mathrm{Fg}$ and its receptor, $\alpha_{\mathrm{IIb}} \beta_{3}$, in the development of the acute phase of NTN. Moreover, the data would appear to resolve the participation of $\mathrm{Fg}$ into two separate components: PLT-PMN coactivation in situ and PLT-PMN comigration into glomeruli.

\section{Methods}

Reagents and materials. Lewis rats were obtained from Charles River Breeding Laboratories (Wilmington, MA). Collagenase type I, trypsin 
type III, EDTA, diaminobenzidine, FITC-conjugated sheep anti-mouse IgG Fab fragments, thrombin, cytochrome $c$, and Ancrod were purchased from Sigma Chemical Co. (St. Louis, MO). PMA was obtained from BIOMOL Research Labs Inc. (Plymouth Meeting, PA). Glycogen was obtained from Fisher Scientific (Fair Lawn, NJ). SC-49992, a peptidomimetic of the $\mathrm{Fg}$ binding sequence RGD, was provided by Searle Pharmaceuticals (Skokie, IL). Hematoxylin was obtained through EM Diagnostic Systems (Gibbstown, NJ). FITC-conjugated goat anti-rat Fg, FITC-conjugated affinity-purified goat anti-rabbit IgG, and FITCconjugated goat anti-rat C3 were purchased from Cappel Laboratories (West Chester, PA). Monoclonal anti-rat leukocyte common antigen (CD45) was purchased from Chemicon International, Inc. (Temecula, CA). Horseradish peroxidase (HRP)-labeled rabbit anti-mouse IgG was purchased from Bio-Rad Laboratories (Richmond, CA). Rabbit anti-rat PMN polyclonal antiserum was purchased from Accurate Chemical \& Scientific Corp. (Westbury, NY).

Monoclonal antibody to $\beta_{3}$ integrin was raised and characterized as previously described (F11) (15). Rabbit anti-rat PLT polyclonal antiserum was raised and characterized also as described previously (6). For visualizing the PLTs in glomerular cell preparations, this antibody was purified using protein A-Sepharose (Pharmacia LKB Biotechnology, Uppsala, Sweden) and directly labeled with HRP using a kit from Zymed Laboratories, Inc. (South San Francisco, CA).

Induction of NTN and its pharmacologic/immunologic manipulation. The preparation of nephrotoxic serum has been detailed previously (6). This antiserum contains no detectable endotoxin $(<0.1 \mathrm{U} / \mathrm{ml})$. To induce nephritis, rats were given $5 \mathrm{ml} / \mathrm{kg}$ intravenously (i.v.). Urine was collected using a metabolic cage, and proteinuria was measured by the Bradford assay (Bio-Rad Laboratories). Glomeruli and renal tissue were harvested 3 or $24 \mathrm{~h}$ after disease induction for the analyses below. Glomeruli were harvested from kidneys perfused free of blood.

Various pharmacologic/immunologic manipulations were performed as follows: to defibrinate animals, Ancrod $50 \mathrm{U} / \mathrm{kg}$ was injected subcutaneously thrice daily for $5 \mathrm{~d}$ before disease induction. Prolonged treatment with Ancrod is necessary to deplete the internal pool of Fg within PLTs (16). PLT depletion was induced before induction of disease by the injection of the aforementioned anti-PLT antiserum $10 \mathrm{ml} / \mathrm{kg}$ which decreases circulating PLT counts to undetectable levels but does not affect circulating leukocyte/PMN counts (reference 6 and data not shown). PMN depletion was generated by injection of anti-PMN antiserum $5 \mathrm{ml} / \mathrm{kg}$. This protocol decreased circulating PMNs by $>90 \%$ but did not affect circulating PLT counts (data not shown).

To effect Fg receptor blockade, animals were given SC-49992, an RGD peptidomimetic $(17,18)$. Although RGD peptides and their mimetics are not generally effective in inhibiting rat PLT aggregation in vitro, some RGD peptide analogues have been shown to prevent collageninduced thrombocytopenia in rats in vivo $(19,20)$. We therefore used a potent mimetic of the RGDF tetrapeptide, SC49992, which has been demonstrated to inhibit human and dog PLT aggregation $(17,18)$ and to prevent collagen-induced thrombocytopenia in the rat $\left(\mathrm{ED}_{50}\right.$ $53 \mu \mathrm{g} / \mathrm{kg}$ ).

We used a variant of the above protocol for induction of NTN because the short half-life of SC-49992 necessitated i.v. infusion. In these experiments, an intraarterial cannula was placed in the femoral artery under anesthesia to allow infusion of SC-49992. Animals were then allowed to recover, during which time they were infused with saline $2 \mathrm{ml} / \mathrm{h}$ to help maintain urine flow. After a 2-h period of equilibration, animals were injected with SC-49992 $1 \mathrm{mg} / \mathrm{kg}$ or an equivalent amount of vehicle (saline) followed by nephrotoxic serum $5 \mathrm{ml} / \mathrm{kg}$. For the next $3 \mathrm{~h}$, animals were either given an infusion of SC-49992 $80 \mu \mathrm{g} /$ $\mathrm{kg}$ per minute in saline or an equivalent volume of saline, at $2 \mathrm{ml} / \mathrm{h}$, and urine was collected. At the end of $3 \mathrm{~h}$, animals were killed as above to obtain glomeruli or tissue for the following analyses.

Glomerular isolation, cell dissociation, and immunostaining. Glomeruli were isolated from saline-perfused kidneys using a sieving protocol described previously which yields a preparation of glomeruli 80 $90 \%$ pure (6). The glomeruli were then dispersed into single cell preparations by a previously published enzymatic dissociation protocol (6).
This protocol yields a single cell suspension of mesangial cells and leukocytes. Occasional endothelial cells are present; however, epithelial cells are largely lost. Cell counts were performed after dissociation to obtain total cell yields and to assess viability (uniformly $>90 \%$ by trypan blue exclusion).

Glomerular leukocytes were quantified in these cell dissociates by immunocytochemistry. Aliquots of cell suspensions ( 30,000 cells) were pelleted onto slides by cytocentrifuge (Shandon Inc., Pittsburgh, PA). The cells were fixed with methanol/acetone (50:50), and leukocytes were labeled with an anti-CD45 antibody as previously detailed (6). Hematoxylin was used as a counterstain. The positive labeled cells were quantified as a percentage of the total by light microscopy and categorized by nuclear morphology as to lineage. Leukocyte counts per glomerulus were then calculated by multiplying leukocyte percentages (or subset fractions) by the total glomerular cell number. This technique correlates well with other methods of leukocyte quantification, such as in situ staining.

A similar protocol was used to assess the presence of PLTs in the glomerular cell dissociates using the aforementioned HRP-labeled antiPLT polyclonal antibody as the detecting reagent (6). PLTs were identified by positive staining as well as appropriate morphology.

For FACS $@$ analysis, $1 \times 10^{6}$ glomerular cells were incubated with anti-rat CD45 or anti-rat $\beta_{3}$ integrin monoclonal antibodies for $45 \mathrm{~min}$ in PBS with $10 \%$ goat serum at $4^{\circ} \mathrm{C}$, washed two times with PBS, and finally incubated with sheep anti-mouse IgG $\mathrm{F}\left(\mathrm{ab}^{\prime}\right)$-FITC for $45 \mathrm{~min}$ at $4^{\circ} \mathrm{C}$. Cells were again washed two times, and the samples were analyzed on a FACScan® (Becton Dickinson, Mountain View, CA).

Glomerular immunohistology. Glomerular depositions of rabbit IgG, rat $\mathrm{C} 3$, and rat $\mathrm{Fg}$ were determined by direct immunofluorescence on fresh frozen tissue using the aforementioned antibodies and previously published protocols (21). Glomerular expression of CD45 or $\beta_{3}$ integrin was visualized in fresh frozen tissue using the appropriate primary antibodies (see above, 1:100 dilution in PBS with 10\% goat serum) along with an avidin-biotin-HRP kit from Vector Labs Inc. (Burlingame, CA). The secondary antibody in the kit was a biotinylated horse anti-mouse IgG. Staining used diaminobenzidine as the HRP substrate and hematoxylin as the counterstain.

PLT-PMN interactions in vitro. PMNs were isolated by peritoneal lavage from rats injected with $7 \mathrm{ml}$ of $1 \%$ glycogen intraperitoneally 4 $\mathrm{h}$ previously (preparations $>95 \%$ pure). PLTs were isolated from blood diluted 1:2 with heparinized saline by centrifugation at $800 \mathrm{~g}$ and washed with HBSS before use. Incubations for the respiratory burst were performed in a volume of $0.4 \mathrm{ml}$ and contained PMNs $\left(2 \times 10^{6}\right)$ without or with PLTs $\left(2 \times 10^{8}\right)$. Cytochrome $c(1 \mathrm{mg} / \mathrm{ml})$ was included in the incubation as an indicator of superoxide production. Incubations were stimulated with either PMA $(1 \mu \mathrm{M})$ or thrombin $(3 \mathrm{U} / \mathrm{ml})$ for $30 \mathrm{~min}$ at $37^{\circ} \mathrm{C}$. In experiments to examine the role of $\mathrm{Fg}$ receptor blockade, SC-49992 $(1 \mathrm{mg} / \mathrm{ml})$ or anti- $\beta_{3}$ monoclonal antibody $(10 \mu \mathrm{g} / \mathrm{ml})$ was preincubated with cells for $15 \mathrm{~min}$ at $37^{\circ} \mathrm{C}$ before stimulation. Superoxide production was measured by spectrophotometry (absorbance at 549 $\mathrm{nm}$ ) and is expressed in relative units normalized for the agonist control (agonist stimulation $=100$ ).

Statistical analysis. Statistical analysis was performed with the aid of STATA (Computing Resource Center, Los Angeles, CA). Values are expressed as mean \pm SEM. Each replicate represents the data from one animal. Two-way comparisons were made with a two-tailed Student's $t$ test (unpaired or paired as appropriate). When comparisons were made between multiple groups, ANOVA was performed along with Bonferroni's correction for multiple comparisons. Significance levels are indicated in the text or figure legends.

\section{Results}

Defibrination and the evolution of NTN. To begin to dissect out the role of $\mathrm{Fg}$ in the development of the inflammatory lesion of NTN, rats were defibrinated with Ancrod for a period of 5 $\mathrm{d}$ before disease induction to deplete both circulating levels of 


\section{Rabbit IgG}

NTN - 3h
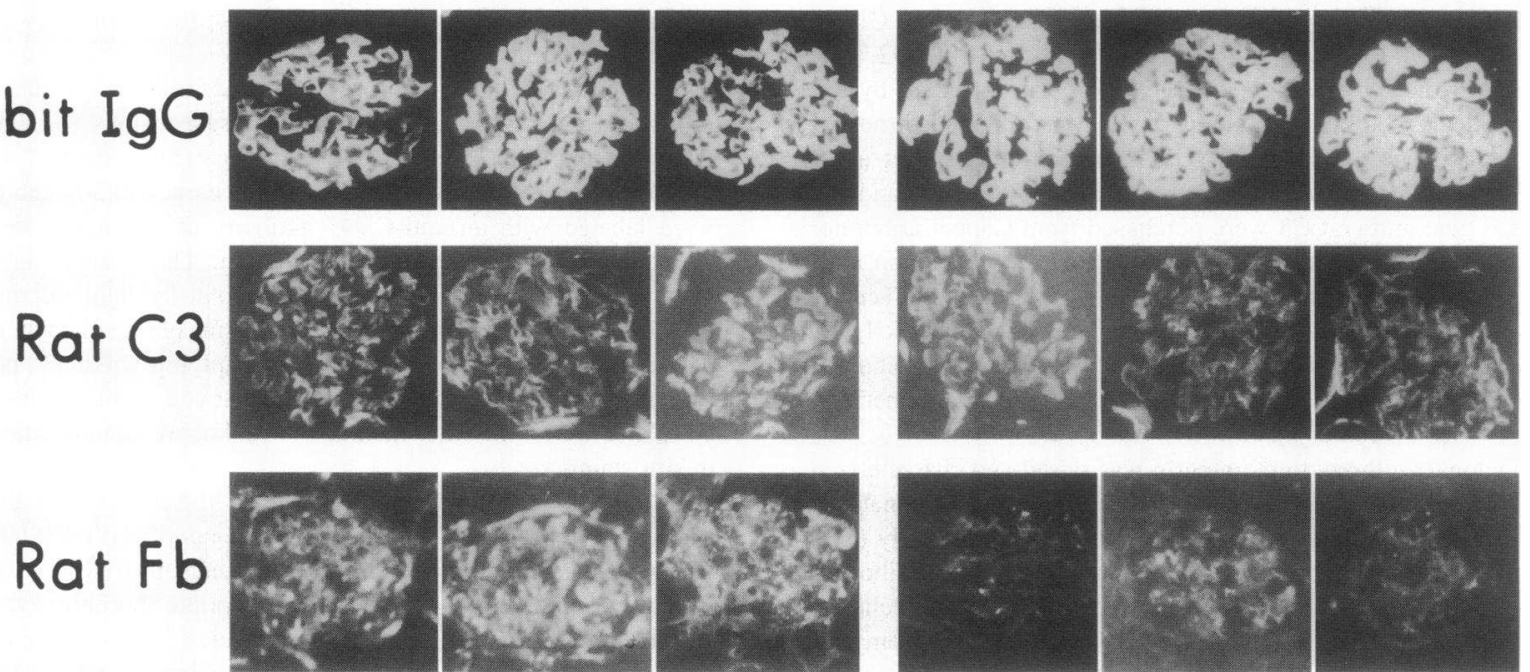

Figure 1. Effect of defibrination on the intraglomerular deposition of heterologous IgG and endogenous $\mathrm{C} 3$ and Fg during NTN. NTN was induced with rabbit polyclonal antibody to rat glomerular basement membrane (GBM) in unmanipulated and defibrinated animals. Deposition of rabbit IgG and rat $\mathrm{C} 3$ and $\mathrm{Fg}$ were detected by direct immunofluorescence on fresh frozen renal tissue obtained $3 \mathrm{~h}$ after disease induction. The figure presents representative glomeruli from a nephritic and a nephritic-defibrinated animal. No staining was evident for rabbit IgG, rat C3, or rat Fg in normal controls (not shown).

Fg as well as the reservoir of Fg within PLTs (16). To evaluate the efficacy of defibrination, we examined the effect of this protocol on the intraglomerular deposition of Fg after induction of NTN. As expected, defibrination markedly attenuated the deposition of $\mathrm{Fg}$ in the glomerulus during the acute phase of nephritis, although there was no substantial effect on the deposition of either rabbit IgG (nephrotoxic serum) or C3 (Fig. 1).

We concomitantly assessed the effect of Fg depletion on the proteinuria which accompanies NTN and which serves as an index of the invasion of inflammatory cells into the glomerulus and their activation (7). Animals given nephrotoxic serum exhibited an approximately 10-fold elevation in urine protein over the $24 \mathrm{~h}$ after disease induction compared with normal controls (Fig. 2). Defibrinated nephritic animals developed substantially less proteinuria than their nephritic counterparts (ap- proximately a $70 \%$ decrease compared with nephritic animals, Fig. 2; Fg comprises $<5 \%$ of the plasma protein content).

Because of the strong correlation between glomerular leukocyte numbers and proteinuria in NTN (7), we next addressed whether the decrease in proteinuria which resulted from defibrination occurred pari passu with a decrease in the influx of leukocytes. As we have found in past studies, NTN was characterized by a marked increase in glomerular leukocytes at 3 and $24 \mathrm{~h}$ : PMNs predominated at the early time point but were replaced by macrophages at $24 \mathrm{~h}$ (Fig. 3). With Fg depletion there was a modest decline in glomerular leukocytes at both time points although the change was not statistically significant (approximately a 25-30\% decrease, Fig. 3). In terms of leukocyte subpopulations, though, Fg depletion significantly diminished the influx of PMNs at $3 \mathrm{~h}$ (by $\sim 50 \%$ ).

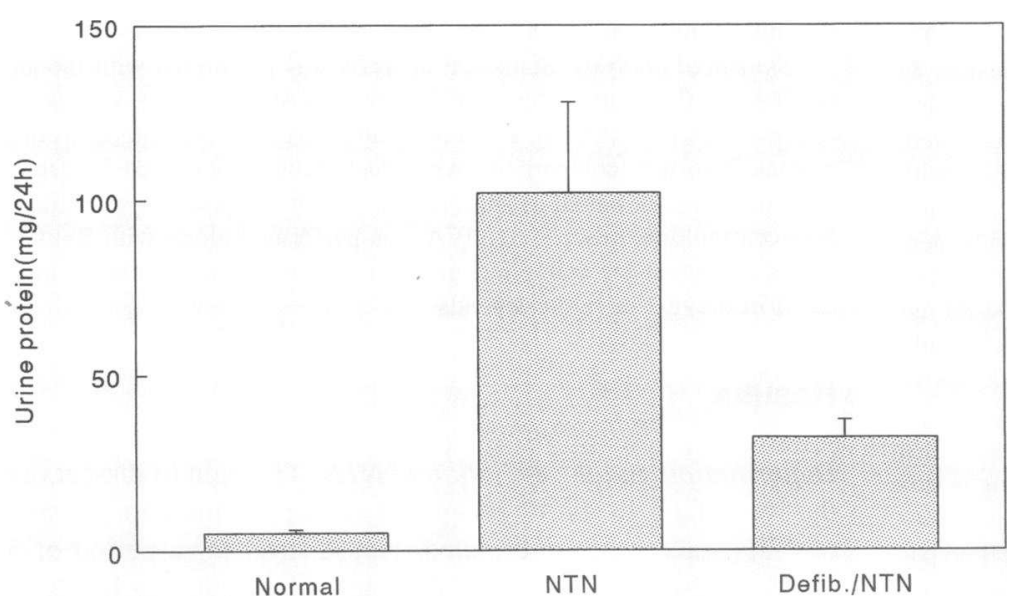

Figure 2. Effect of defibrination on proteinuria in NTN. NTN was induced with a rabbit polyclonal antibody to rat GBM in unmanipulated and defibrinated animals. Nondisease controls were also assessed. Urine was collected for $24 \mathrm{~h}$ after disease induction, and the protein content was calculated by a microspectrophotometric assay. $n=3,5$, and 6 for the three groups shown (left to right: normal, nephritic, nephritic/defibrinated). The difference between nephritic and defibrinated-nephritic animals was significant $(P<0.05)$. 
A

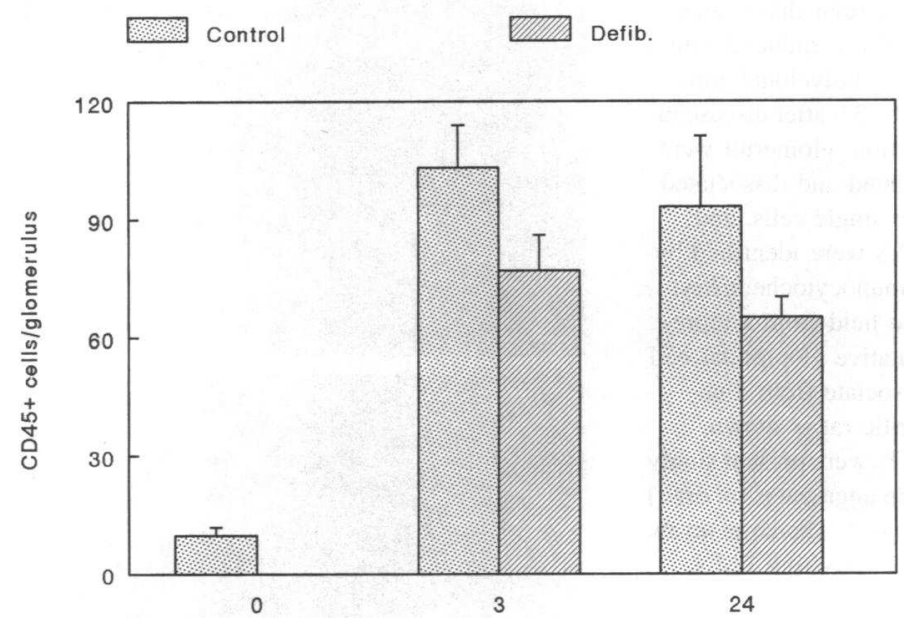

B
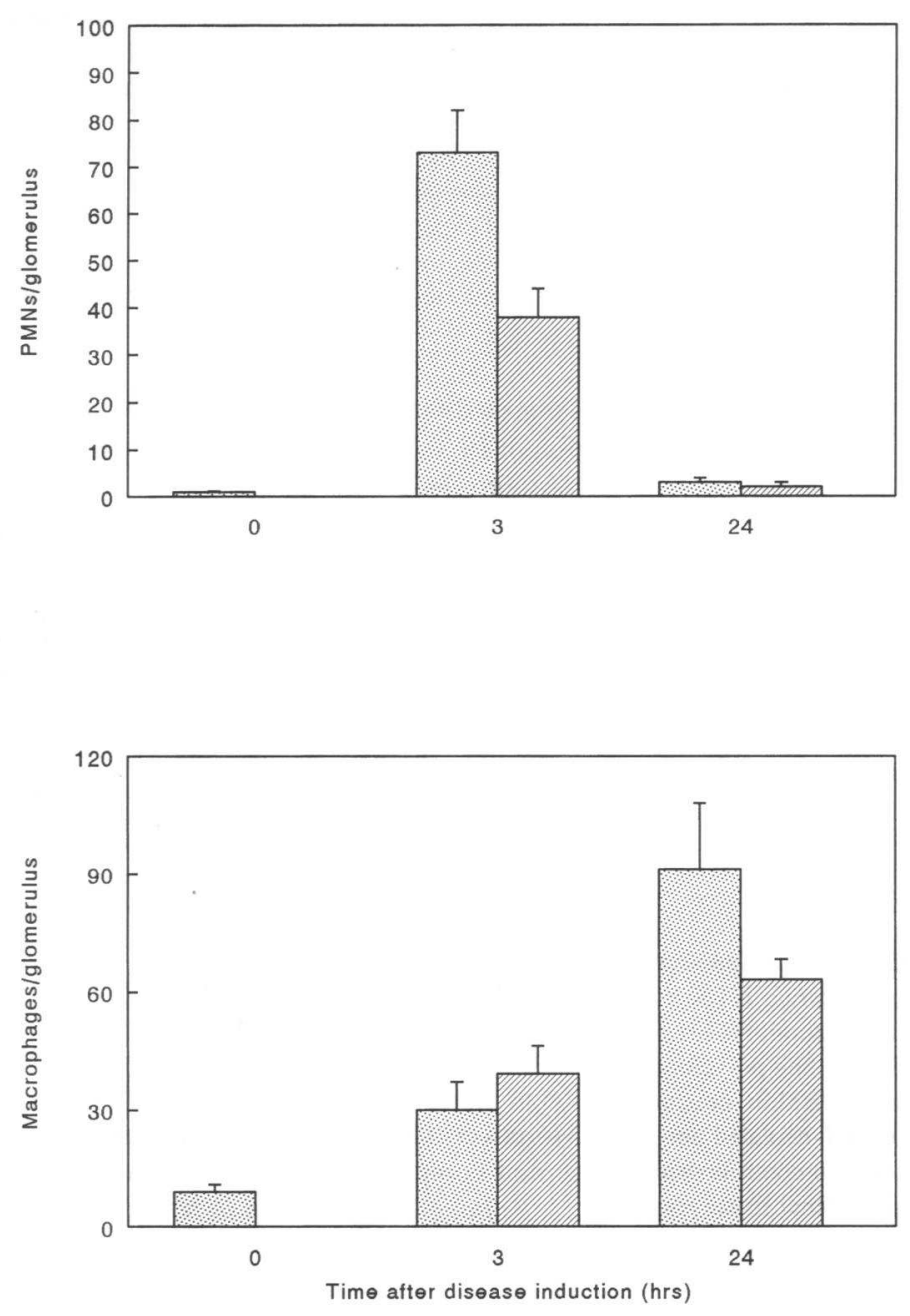

Figure 3. Effect of defibrination on leukocyte influx into the glomeruli in NTN. NTN was induced with a rabbit polyclonal antibody to rat GBM in unmanipulated and defibrinated animals. Nondisease controls were also assessed. Glomeruli were isolated at 0,3 , or $24 \mathrm{~h}$ after disease induction and dissociated into single cells, and leukocytes were quantified and subtyped by immunocytochemical staining. $n=3,6,7,5$, and 6 for the five groups shown (left to right: normals, nephritic at $3 \mathrm{~h}$ after disease induction, nephritic/defibrinated at $3 \mathrm{~h}$ after disease induction, nephritic at $24 \mathrm{~h}$ after disease induction, and nephritic/defibrinated at $24 \mathrm{~h}$ after disease induction). $A$, total leukocytes/glomerulus; $B$, PMNs/glomerulus. The difference between nephritic and defibrinated-nephritic animals for glomerular PMN counts at $3 \mathrm{~h}$ was significantly different $(P<0.05) . C$, macrophages/glomerulus.

Since a cooperative interaction between PLTs and PMNs is critical to the efficient influx of PMNs into the glomerulus (6), we additionally quantified the PLT content of glomerular cell dissociates. As shown in Fig. 4, dissociates from nephritic glomeruli contained substantial numbers of PLTs, singly or in aggregates, both cell-associated and non-cell-associated. PLTs 


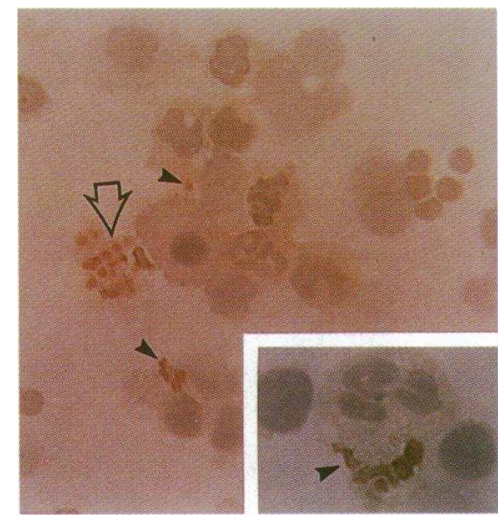

Figure 4. PLT content of glomerular dissociates. NTN was induced with a rabbit polyclonal antibody. $3 \mathrm{~h}$ after disease induction, glomeruli were isolated and dissociated into single cells, and PLTs were identified by immunocytochemistry. One field from a representative glomerular cell dissociate from a nephritic rat is shown. PLTs were present singly or in aggregates (arrows) both in close proximity to cells or non-cell associated. The large arrow shows a clump of PLTs. Inset, PLTs in close proximity to a PMN.

associated with PMNs were also noted (Fig. 4, inset). Defibrination moderately decreased the apparent numbers of PLTs: $18.0 \pm 0.5$ vs $11.0 \pm 2.0$ PLTs $/ 100$ cells in nephritic and defibrinated nephritic animals, respectively $(n=3, P=0.07)$.

What was notable about these results taken together was the relative discrepancy between the decrease in total leukocyte and PLT influx with Fg depletion $(\sim 25-40 \%)$ and the more substantial decrease in proteinuria $(\sim 70 \%)$. This discrepancy
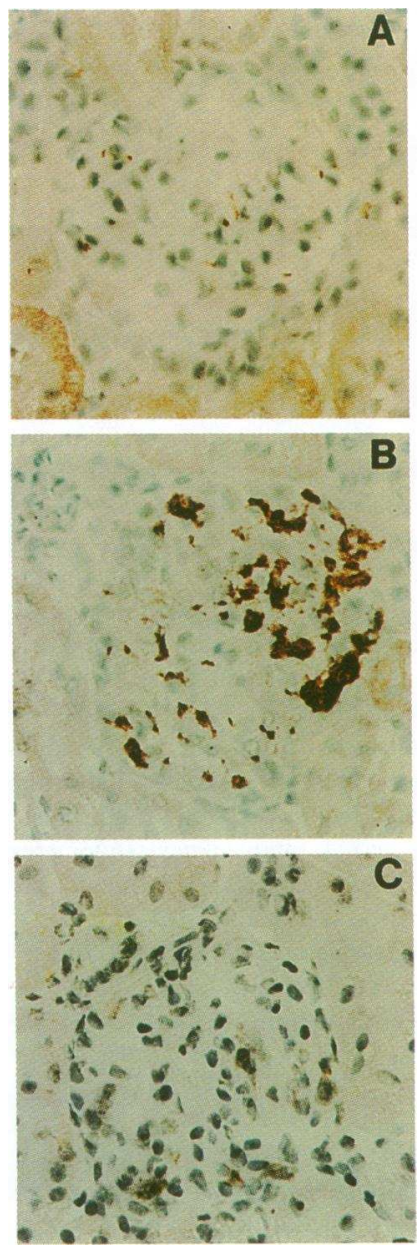
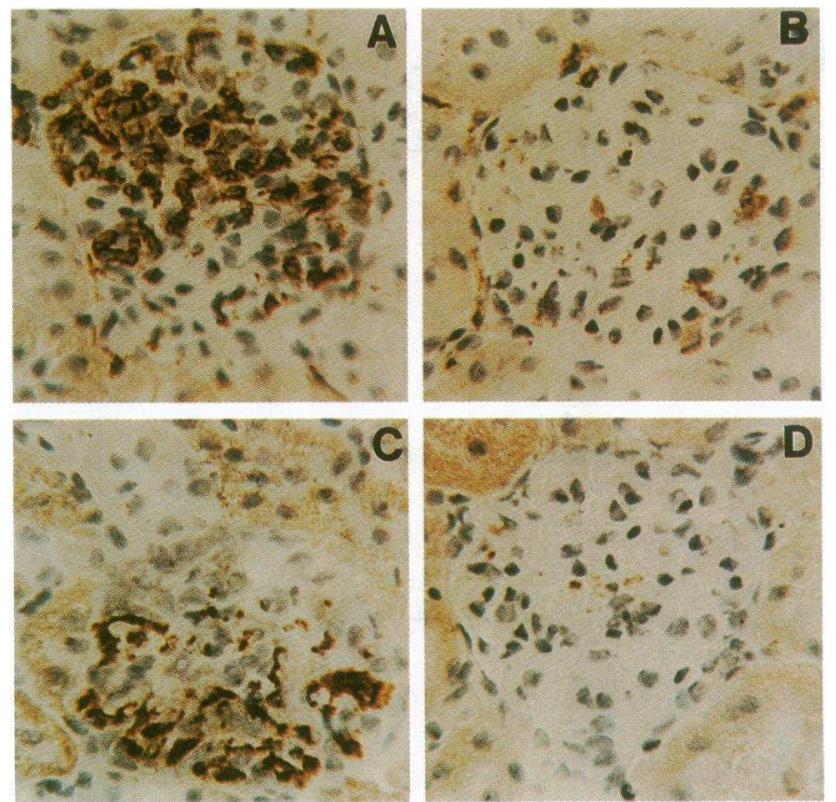

Figure 7. Glomerular expression of $\beta_{3}$ integrins during NTN: effect of PMN depletion. NTN was induced with a rabbit polyclonal antibody to rat GBM in unmanipulated and PMN-depleted animals. Nondisease controls were also assessed. Renal tissue was obtained $3 \mathrm{~h}$ after disease induction, and the expression of CD45 and $\beta_{3}$ integrins was determined by immunohistochemistry. Representative glomeruli from one of three identical experiments are shown. $A$ and $C, \mathrm{CD} 45$ and $\beta_{3}$ integrin expression, respectively, in a nephritic animal; $B$ and $D$, CD45 and $\beta_{3}$ integrin expression, respectively, in a PMN-depleted nephritic animal.

suggested that Fg might be more important to the in situ activation of PLTs and PMNs than their emigration from the vasculature into the glomerulus.

Effect of Fg receptor blockade on NTN. We were able to address this issue using the peptidomimetic of the RGD binding motif of Fg, SC-49992, which blocks Fg binding to PLT $\alpha_{\mathrm{Im}} \beta_{3}$ $(17,18)$. Because of the short half-life of this compound, we evaluated its effects when administered over the $3 \mathrm{~h}$ after disease induction. Similar to Fg depletion, PLT Fg receptor blockade largely attenuated the proteinuria which occurred in NTN (by $\sim 90 \%$, Fig. 5). However, in contrast to Fg depletion, SC-49992 had little effect on either total leukocyte or PMN influx into glomeruli at $3 \mathrm{~h}(<10 \%$ decrease, Fig. 5). We did observe a tendency towards a decline in PLT counts within glomerular cell dissociates with SC-49992: $20.0 \pm 2.0$ vs $13.0 \pm 2.0$ PLTs/100 cells in nephritic and SC-49992-treated nephritic animals, respectively, though this was not statistically significant $(n=3, P=0.18)$.

These data reinforced the impression that $\mathrm{Fg}$ was critical to the in situ activation of PLTs and PMNs within the glomerulus. Moreover, they suggested that expression of $\alpha_{\mathrm{IIb}} \beta_{3}$ was an essential component of this coactivation response.

$\beta_{3}$ integrin expression in NTN. To understand this issue, we next addressed the expression of $\beta_{3}$ integrins within glomeruli during the acute phase of NTN. In normal animals, there was no evident expression of $\beta_{3}$ integrins by glomerular cells, although there were occasional small positively staining cellular fragments (presumably PLTs) within the vasculature (Fig. $6 \mathrm{~A}$ ). By $3 \mathrm{~h}$ after disease induction, however, expression of $\beta_{3}$ integrins was dramatically increased in the glomerulus (Fig. $6 \mathrm{~B}$ ). Our initial presumption was that this increased expression was 

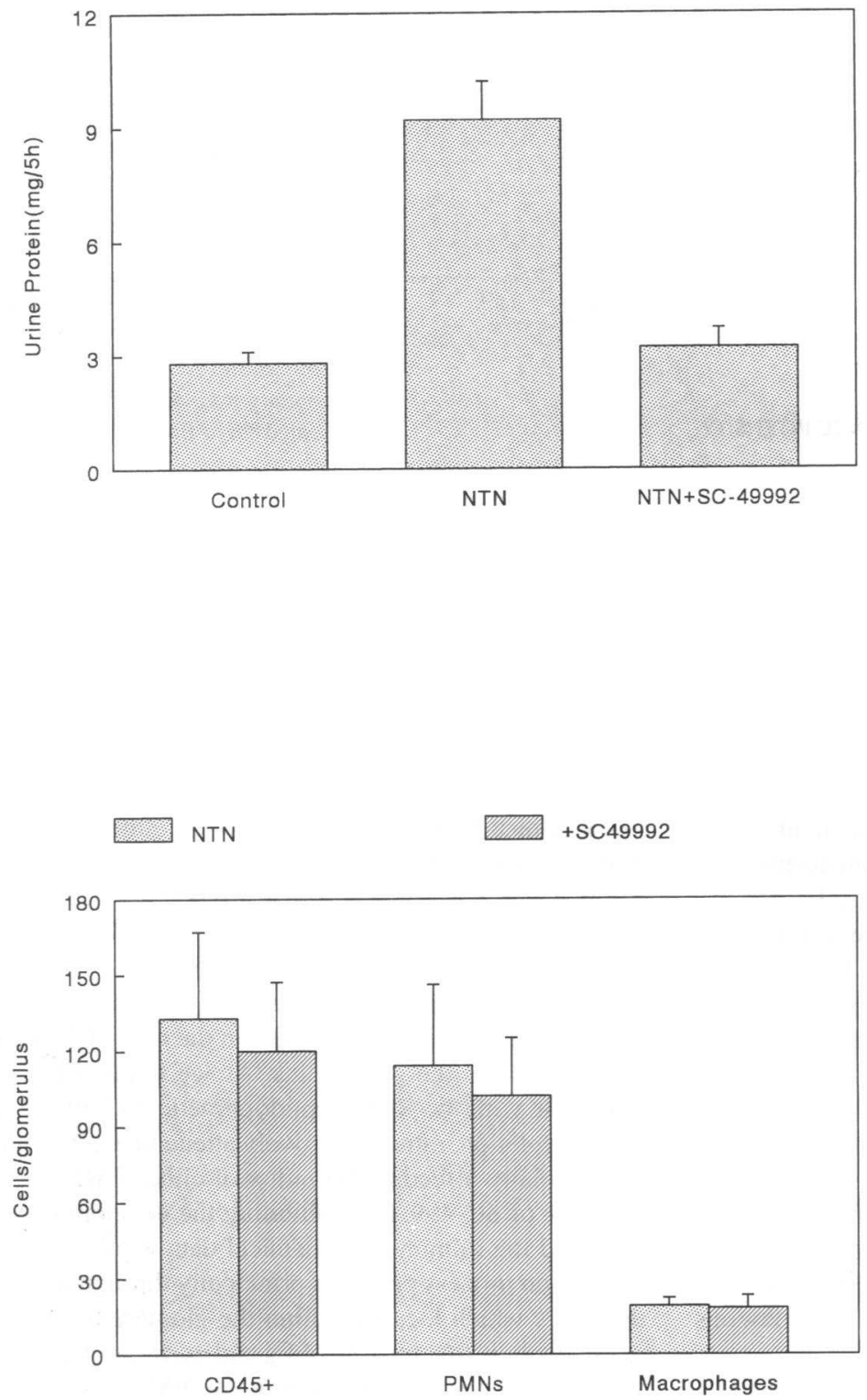

Figure 5. Effect of SC-49992 on NTN. NTN was induced with a rabbit polyclonal antibody to rat GBM in animals infused with either SC-49992 or saline (i.e., vehicle) intravenously. Nondisease controls were also assessed. Urine was collected for a period of $3 \mathrm{~h}$ for determination of proteinuria. Glomeruli were then isolated from nephritic animals (with or without SC-49992) and dissociated into single cells, and leukocytes were quantified and subtyped by immunocytochemical staining. $A$, proteinuria in normal controls, nephritic animals, and nephritic animals treated with SC-49992 $(n=4,7$, and 6, respectively). Proteinuria was significantly different in nephritic animals relative to the other two groups $(P<0.05)$. B, leukocyte content (total, PMNs, and macrophages) of glomeruli from nephritic animals and nephritic animals treated with SC-49992. $n=4$ for both.

a function of PLT deposition, and, in consequence, we subsequently examined the effect of PLT depletion on the increase in $\beta_{3}$ integrin with NTN. As shown in Fig. $6 C$, PLT depletion completely attenuated the increase in $\beta_{3}$ integrin expression.

However, in light of our previous results which show a cooperative interaction between PMNs and PLTs with respect to migration (6), we additionally addressed the effect of PMN depletion on $\beta_{3}$ integrin expression within glomeruli. As shown in Fig. 7, $A$ and $B$, PMN depletion markedly attenuated the influx of PMNs into the glomerulus as exhibited by staining for CD45. Moreover, PMN depletion, like PLT depletion, also completely prevented the increase in $\beta_{3}$ integrin expression in acute NTN (Fig. 7, $C$ and $D$ ).

These immunohistologic findings demonstrated that the increased glomerular $\beta_{3}$ integrin expression was a function of both PLTs and PMNs but did not definitively address alterations in expression at a cellular level. We broached this issue by performing FACS $®$ on glomerular cell dissociates using our anti- $\beta_{3}$ integrin monoclonal. As shown in Fig. $8 A$, numerous CD45-positive cells were present in inflamed glomeruli. However, staining of the identical preparation with the anti $-\beta_{3}$ integrin monoclonal showed a small population of positively staining cells of varying intensity. Using immunocytochemistry we were able to identify the relatively more intensely stained particles as PLTs which were both free and adherent to other cells similar to that shown in Fig. 4 (data not shown). $\beta_{3}$ integrin expression by PMNs or mesangial cells was modest by immunocytochemistry and no alteration in staining was evident with nephritis with this method (data not shown).

Although defibrination markedly diminished proteinuria, $\beta_{3}$ integrin expression was not noticeably affected by this manipulation (data not shown). This result is consistent with our aforementioned observations that defibrination only modestly altered PLT influx. 
$\mathbf{A}$

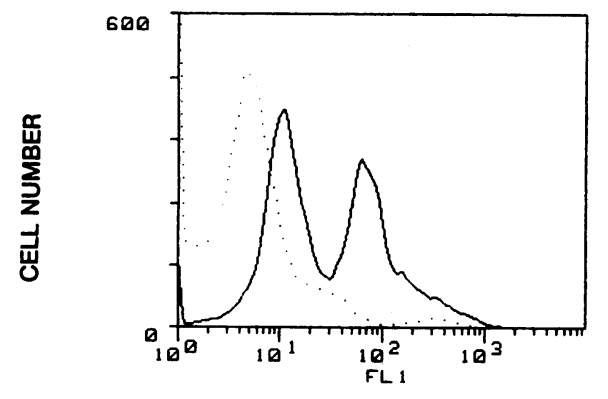

B

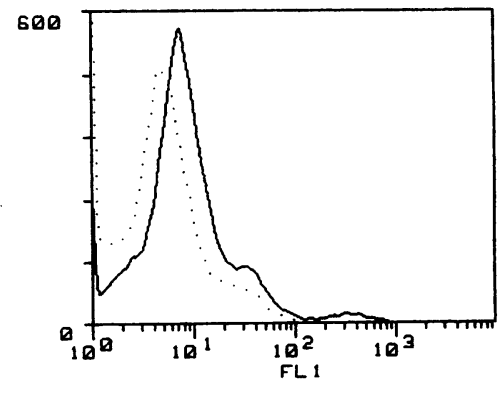

Figure 8. CD45 and $\beta_{3}$ integrin expression in dissociated glomerular cells from inflamed glomeruli. Glomeruli from nephritic animals ( $3 \mathrm{~h}$ after disease induction) were dissociated into single cells, and CD45 $(A)$ and $\beta_{3}$ integrin expression $(B)$ were determined by FACS $\otimes$. Solid lines represent the inclusion of primary antibody; dotted lines represent its omission (i.e., nonspecific binding of the secondary antibody). This experiment was repeated three times with similar results.
$F g$ and $P L T-P M N$ activation in vitro. These in vivo studies suggested that Fg contributed to glomerular damage in NTN (as manifested by proteinuria) via the upregulated expression of $\alpha_{\mathrm{IIb}} \beta_{3}$ which resulted from PLT-PMN influx into glomeruli. To understand in more specific terms how $\mathrm{Fg}$ might contribute to proteinuria, we examined PLT-PMN activation in vitro and the effect of Fg receptor blockade on this process. We focused on the PMN respiratory burst given prior studies implicating this process as a proximate cause of proteinuria in immunemediated glomerular injury (22), and given data suggesting that Fg can mediate PLT-PMN coactivation in vitro $(23,24)$.

PMNs incubated alone or with thrombin were quiescent in terms of the respiratory burst (Fig. $9 A$ ). When PLTs and PMNs were coincubated, a modest increase in the respiratory burst was noted (possibly in part resulting from PLT activation during isolation, Fig. $9 A$ ). However, when thrombin was added to PLT-PMN coincubations, a threefold increase in superoxide formation was seen (Fig. $9 \mathrm{~A}$ ). This stimulatory effect of thrombin in PLT-PMN coincubations was inhibited by $\sim 50 \%$ by Fg receptor blockade (Fig. $9 \mathrm{~A}$ ). Of interest, although $\mathrm{Fg}$ receptor blockade consistently inhibited PLT-PMN coactivation in terms of the respiratory burst, we noted that SC-49992 (but not anti- $\beta_{3}$ antibody) inhibited the visually apparent heterologous aggregation.

PMA, in contrast to thrombin, directly stimulated the PMN respiratory burst (Fig. $9 \mathrm{~B}$ ). No further enhancement of this response was seen when platelets were added to the incubation (data not shown). SC-49992 modestly inhibited this response $(\sim 25 \%)$, whereas anti- $\beta_{3}$ antibody had little effect (Fig. $9 B$ ).

\section{Discussion}

PMN-PLT interactions appear to play a critical role in the development of the acute inflammatory response and the resultant tissue damage and dysfunction $(8,9)$. In our previous study and this current work, we have specifically corroborated this hypothesis within the context of immune complex-mediated glomerulonephritis. As we have observed, PLTs and PMNs colocalize to the glomerulus in the acute phase of NTN and become coordinately activated, resulting in glomerular dysfunction and a marked change in glomerular eicosanoid production (6). The intent of the current work was to begin to delineate the molecular mechanisms which potentially mediate both the localization of these cells to the glomerulus and their activation in situ.
This study would suggest that $\mathrm{Fg}$ plays an important role in both of these processes in that defibrination substantially attenuated the acute glomerular damage in NTN (as manifested by proteinuria) and significantly, though less substantially, decreased the accompanying influx of PMNs and PLTs into glomeruli. These results corroborate and expand on earlier work which showed that defibrination or induction of fibrinolysis could ameliorate immune complex-mediated glomerulonephritis both histologically and functionally although this effect was not invariable $(10,11)$. The variability seen in these earlier studies may have related in part to the persistence of $\mathrm{Fg}$ within PLTs (16), an issue which we circumvented by pretreating animals for $5 \mathrm{~d}$ before disease induction.

It would appear from the current data that the major role of Fg in acute NTN is to participate in the PLT-PMN coactivation process within the glomerulus. As we found, PLT Fg receptor blockade with the RGD peptidomimetic, SC-49992, in contrast to Fg depletion, rather selectively attenuated proteinuria without substantially affecting the influx of either PMNs or PLTs. The efficacy of SC-49992 in inhibiting the development of proteinuria and the immunocytochemical data would suggest that this activation process proceeds principally through $\alpha_{\mathrm{IIb}} \beta_{3}$ expressed by PLTs which localize within the glomerulus. We would further conclude that the role of $\mathrm{Fg}$ and $\alpha_{\mathrm{IIb}} \beta_{3}$ in glomerulonephritis is to mediate PLT activation of the PMN respiratory burst in situ and thereby proteinuria. As we found in our in vitro studies, Fg receptor blockade attenuated PLT-dependent PMN production of reactive oxygen species, reactive oxygen species being one of the proximate causes of proteinuria in acute immunemediated glomerulonephritis (22).

Our conclusions regarding the role of $\mathrm{Fg}$ and $\alpha_{\mathrm{mb}} \beta_{3}$ in NTN, however, are subject to the caveat that SC-49992 is not absolutely specific for the PLT Fg receptor $\alpha_{\mathrm{IIb}} \beta_{3}$. SC-49992 can block other receptors recognizing the RGD motif such as the vitronectin receptor $\left(\alpha_{v} \beta_{3}\right)$ or the leukocyte response integrin (another member of the $\beta_{3}$ integrin family), albeit less well (references 17, 18, and 25-27, and Gresham, H., personal communication). We, in fact, noted that even the direct activation of the PMN respiratory burst with PMA could be partially inhibited with SC-49992, implying that the aforementioned $\beta_{3}$ integrins on PMNs may also participate in the proinflammatory effects of $\mathrm{Fg}$ in glomerulonephritis.

PLT-PMN interactions can be bi-directional, though PLTmediated PMN activation and PMN-mediated PLT activation both appear to require surface contact and to be mediated by 


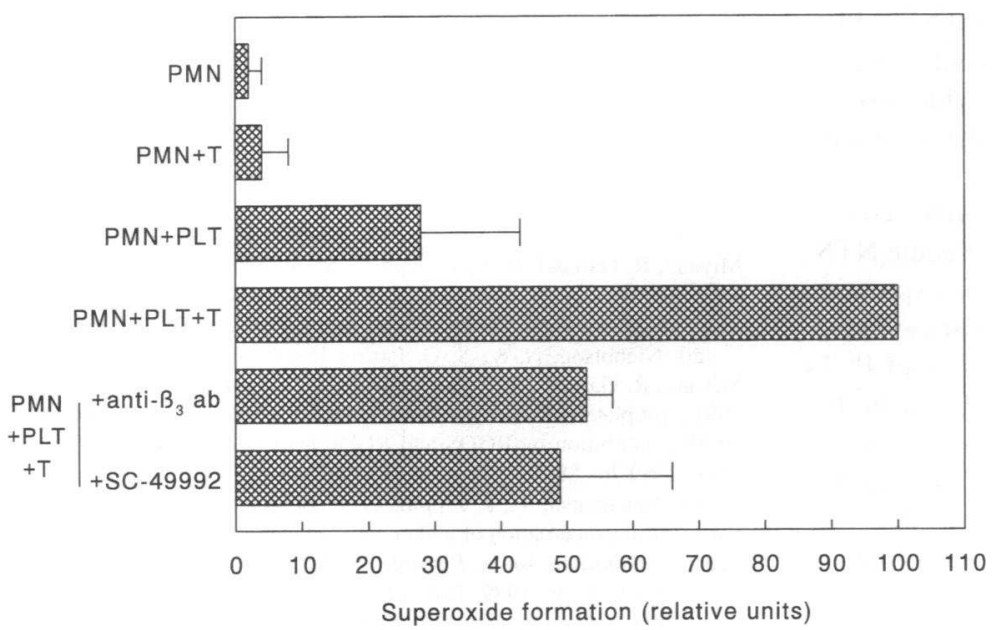

B

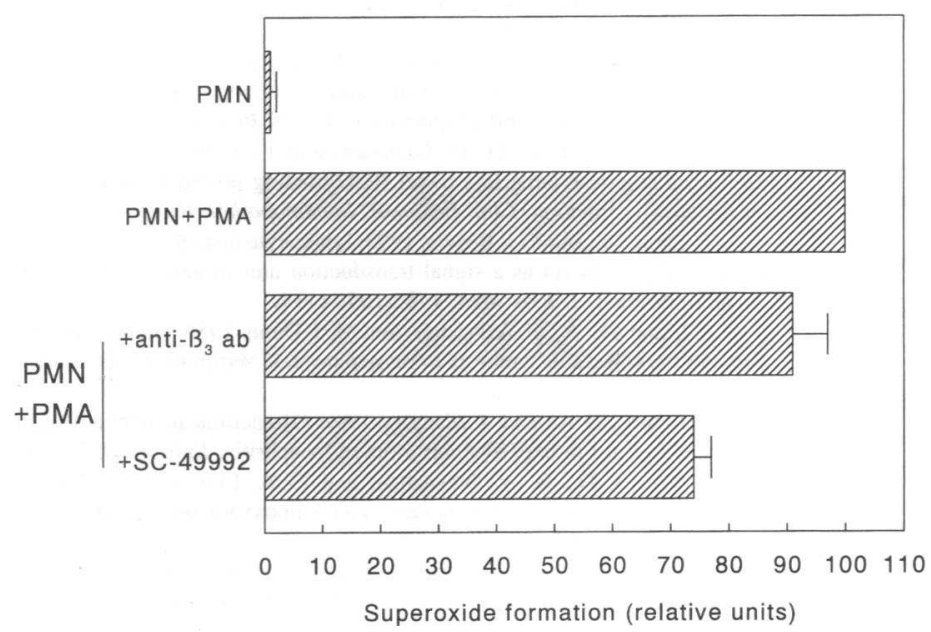

Figure 9. PLT-PMN activation in vitro: role of $\mathrm{Fg}$ and $\beta_{3}$ integrins. PMNs were incubated alone or in the presence of PLTs. Thrombin ( $T$ ) or PMA was added as an agonist. To block the Fg receptor, cells were preincubated with SC-49992 or anti- $\beta_{3}$ antibody before stimulation. The PMN respiratory burst was measured as superoxide formation quantified using cytochrome $c$. Results are presented as arbitrary units relative to agonist stimulation $(=100) . A$, thrombin-stimulated PMN-PLT coincubations. All groups were significantly less than thrombin-stimulated PLTS + PMNs $(P<0.05)$, except for PLTS + PMNs + thrombin +SC-49992 for which $P$ $=0.09 . n=3$ for all groups. $B$, PMA-stimulated PMN incubations. SC-49992 treatment significantly inhibited PMA-stimulated PMN superoxide production ( $P<0.05, n=4$ for all groups). PMA-stimulated PMN superoxide formation was approximately twofold greater than thrombin-stimulated PMNPLT coincubation superoxide formation.
Fg $(23,24)$. In light of the marked increase in glomerular lipid mediator production in the context of NTN $(28-30)$, it is further noteworthy that lipid autacoids may be involved in this reciprocal interaction. Thromboxane may stimulate PLTs to activate PMNs, leukotriene $B_{4}$ may stimulate PMNs to activate PLTs, and PMN-derived platelet activating factor may serve to activate PLTs $(23,24)$. However, with respect to NTN, prior studies would suggest that platelet activating factor, thromboxane, and leukotriene $\mathrm{B}_{4}$ independently play modest roles in the coactivation process $(21,31,32)$.

Although Fg appears to be critical to PLT-PMN coactivation, the present data also suggest that $\mathrm{Fg}$ may play a role in PMN-PLT comigration into glomeruli, albeit a relatively more limited one. Hypothetically, Fg may act as a ligand for PMN $\beta_{2}$ integrins or intercellular adhesion molecule and facilitate their transendothelial migration and/or Fg may serve as a molecular bridge between PLTs and PMNs (13). It is worth noting that $\mathrm{Fg}$ binding to CD11b/CD18 is not mediated by the RGD motif (13) and thus would not be blocked by SC-49992.

However, it is clear that Fg-independent mechanisms are important for the influx of PMNs and PLTs into glomeruli in
NTN. Of relevance to this comment, we observed that PLTPMN aggregation in vitro was not invariably inhibited by $\mathrm{Fg}$ receptor blockade even though the respiratory burst was. Based on the available data, we would surmise that P-selectin/GMP140 might be important to this process $(33,34)$, though we have not been able to substantiate this conclusion using the anti-P selectin monoclonal antibody, PB1.3 (kindly provided by $\mathrm{J}$. Paulson, Cytel, San Diego, CA) (references 35 and 36, and $\mathrm{Wu}, \mathrm{X}$., and J. B. Lefkowith, unpublished data). However, it is possible that other molecules, such as complement components (i.e., C3b and C3bi), might serve to promote PLT-PMN adhesion, bypassing P-selectin $(8,9)$. Understanding the mechanisms by which PLTs adhere to PMNs as they emigrate from the vasculature into the mesangium will require further investigation.

Although the mechanisms of concern for the current study deal with events which occur early in the inflammatory cascade, acute events clearly have important ramifications for chronic outcomes. Within the context of NTN, studies have shown that the magnitude of the acute insult to the glomerulus, if sufficiently severe, can result in glomerulosclerosis (37). Moreover, 
PLTs contain a panoply of mediators which may contribute to perpetuation of the inflammatory response and contribute to the process of glomerulosclerosis such as PDGF, FGF, TNF $\alpha$, PF4, and TGF $\beta$ (37-40). Thus, developing an understanding of the molecular mechanisms operative early on may provide insights into strategies which may be useful to prevent the long-term adverse outcome of glomerular scarring.

In conclusion, $\mathrm{Fg}$ appears to play a critical role in the coactivation of PLTs and PMNs within the glomerulus in acute NTN. This activation is most likely mediated via $\alpha_{\mathrm{II}} \beta_{3}$, the expression of which is markedly increased in glomeruli after disease induction. This altered expression appears due to the influx of PLTs in a PMN-dependent fashion which in part is mediated by Fg, but which clearly is dependent to a large degree on other molecular interactions which remain to be determined. Developing an understanding of these acute events may help in the development of strategies not only to ameliorate acute glomerular injury, but also to prevent glomerular scarring which may result from the initial insult.

\section{Acknowledgments}

This work was supported by National Institutes of Health grant DK37879. Dr. Lefkowith is a Burroughs-Wellcome Scholar in Clinical Pharmacology. Dr. Horton is supported by the Imperial Cancer Research Fund (U.K.).

\section{References}

1. Couser, W. G. 1990. Mediation of immune glomerular injury. J. Am. Soc. Nephrol. 1:13-29.

2. Couser, W. G. 1993. Pathogenesis of glomerulonephritis. Kidney Int. 44:S19-S26

3. Wu, X., J. Pippin, and J. B. Lefkowith. 1993. Attenuation of immunemediated glomerulonephritis with an anti-CD11b monoclonal antibody. Am. J. Physiol. 264:F715-F721.

4. Mulligan, M. S., K. J. Johnson, R. F. Todd III, T. B. Issekutz, M. Miyasaka, T. Tamatani, C. W. Smith, D. C. Anderson, and P. A. Ward. 1993. Requirements for leukocyte adhesion molecules in nephrotoxic nephritis. J. Clin. Invest. 91:577587.

5. Wu, X., A. J. Wittwer, L. S. Carr, B. A. Crippes, J. E. DeLarco, and J. B Lefkowith. 1994. Cytokine-induced neutrophil chemoattractant mediates neutrophil influx in immune complex glomerulonephritis in rat. J. Clin. Invest. 94:337344.

6. Wu, X., J. Pippin, and J. B. Lefkowith. 1993. Platelets and neutrophils are critical to the enhanced glomerular arachidonate metabolism in acute nephrotoxic nephritis in rats. J. Clin. Invest. 91:766-773.

7. Lefkowith, J. B., T. Nagamatsu, J. Pippin, and G. F. Schreiner. 1991 Role of leukocytes in metabolic and functional derangements of experimental glomerulonephritis. Am. J. Physiol. 261:F213-F220.

8. Faint, R. W. 1992. Platelet-neutrophil interactions: their significance. Blood Rev. 6:83-91.

9. Henson, P. M. 1990. Interactions between neutrophils and platelets. Lab. Invest. 62:391-393.

10. Mathieson, P. W., S. Thiru, D. K. Peters, and D. B. G. Oliveira. 1991 Effects of ancrod and rtPA on fibrin accumulation, glomerular inflammation and renal function in nephrotoxic nephritis. Int. J. Exp. Pathol. 72:679-693.

11. Tipping, P. G., N. M. Thomson, and S. R. Holdsworth. 1986. A comparison of fibrinolytic and defibrinating agents in established experimental glomerulonephritis. Br. J. Exp. Pathol. 67:481-491.

12. Hynes, R. O. 1992. Integrins: versatility, modulation, and signaling in cell adhesion. Cell. 69:11-25.

13. Altieri, D. C. 1993. Coagulation assembly on leukocytes in transmembrane signaling and cell adhesion. Blood. 81:569-579.

14. Tang, L., and J. W. Eaton. 1993. Fibrin(ogen) mediates acute inflammatory responses to biomaterials. J. Exp. Med. 178:2147-2156.

15. Helfrich, M. H., S. A. Nesbitt, and M. A. Horton. 1992. Integrins on rat osteoclasts: characterization of two monoclonal antibodies (F4 and F11) to rat beta-3. J. Bone Miner. Res. 7:345-351.

16. Handgama, P. J., M. A. Shuman, and D. F. Bainton. 1990. In vivo defibrination results in markedly decreased amounts of fibrinogen in rat megakaryocytes and platelets. Am. J. Pathol. 137:1393-1399.
17. Nicholson, N. S., S. G. Panzer-Knodle, L. W. King, B. B. Taite, B. T. Keller, F. S. Tjoeng, V. W. Engleman, T. D. Giorgio, and L. P. Feigen. 1994 SC-49992: a potent and specific inhibitor of platelet aggregation. Thromb. Res. 74:523-535

18. Feigen, L. P., N. S. Nicholson, L. W. King, J. G. Campion, F. S. Tjoeng, and S. G. Panzer-Knodle. 1993. SC-49992, a mimetic of the peptide arginineglycine-aspartic acid-phenylalanine that blocks platelet aggregation, enhances recombinant tissue plasminogen activator-induced thrombolysis and prevents reocclusion in a canine model of coronary artery thrombosis. J. Pharmacol. Exp. Ther. 267:1191-1197.

19. Nicholson, N. S., S. G. Panzer-Knodle, A. K. Salyers, B. B. Taite, M Miyano, R. Garland, R. J. Gorczynski, M. Williams, M. Zupec, S. Tsoeng, et al 1991. In vitro and in vivo effects of a non-peptide analog (SC-47643) of RGD as an antiplatelet and antithrombotic agent. Thromb. Res. 62:567-578.

20. Nicholson, N. S., S. G. Panzer-Knodle, A. K. Salyers, B. B. Taite, M. Miyano, R. Garland, R. J. Gorczynski, M. Williams, M. Zupec, S. Tsoeng, et al. 1991. Antiplatelet and antithrombotic effects of platelet glycoprotein IIb/IIIa (GP IIb/IIIa) inhibition by RGDS and RGD(O-me)Y (SC48749). J. Pharmacol. Exp. Ther. 256:876-882.

21. Nagamatsu, T., J. Pippin, G. F. Schreiner, and J. B. Lefkowith. 1992. Paradoxical exacerbation of leukocyte-mediated glomerulonephritis with cyclooxygenase inhibition. Am. J. Physiol. 263:F228-F236.

22. Shah, S. V. 1989. Role of reactive oxygen metabolites in experimental glomerular disease. Kidney Int. 35:1093-1106.

23. Ruf, A., R. F. Schlenk, A. Maras, E. Morgenstern, and H. Patscheke. 1992. Contact-induced neutrophil activation by platelets in human cell suspensions and whole blood. Blood. 80:1238-1246.

24. Zhou, W. G., M. A. Javors, and M. S. Olson. 1992. Platelet-activating factor as an intercellular signal in neutrophil-dependent platelet activation. $J$. Immunol. 149:1763-1769.

25. Gresham, H. D., J. L. Goodwin, P. M. Allen, D. C. Anderson, and E. J. Brown. 1989. A novel member of the integrin receptor family mediates Arg-GlyAsp-stimulated neutrophil phagocytosis. J. Cell Biol. 108:1935-1943.

26. Carreno, M. P., H. D. Gresham, and E. J. Brown. 1993. Isolation of leukocyte response integrin-a novel RGD-binding protein involved in regulation of phagocytic function. Clin. Immunol. Immunopathol. 69:43-51.

27. Zhou, M., and E. J. Brown. 1993. Leukocyte response integrin and integrinassociated protein act as a signal transduction unit in generation of a phagocyte respiratory burst. J. Exp. Med. 178:1165-1174.

28. Lianos, E. A., G. A. Andres, and M. J. Dunn. 1983. Glomerular prostaglandin and thromboxane synthesis in rat nephrotoxic serum nephritis. J. Clin. Invest. 72:1439-1448.

29. Lianos, E. A., and A. Zanglis. 1990. Glomerular platelet-activating factor levels and origin in experimental glomerulonephritis. Kidney Int. 37:736-740.

30. Wu, S. H., B. A. Bresnahan, and E. A. Lianos. 1993. Hemodynamic role of arachidonate 12-lipoxygenases and 5-lipoxygenases in nephrotoxic serum nephritis. Kidney Int. 43:1280-1285.

31. Rovin, B. H., J. B. Lefkowith, and G. F. Schreiner. 1990. Mechanisms underlying the anti-inflammatory effects of essential fatty acid deficiency in experimental glomerulonephritis: inhibited release of a glomerular chemoattractant. $J$. Immunol. 145:1238-1245.

32. Yared, A., C. Albrightson-Winslow, D. Griswold, K. Takahashi, A. Fago, and K. F. Badr. 1991. Functional significance of leukotriene $B_{4}$ in normal and glomerulonephritic kidneys. J. Am. Soc. Nephrol. 2:45-56.

33. Hamburger, S. A., and R. P. McEver. 1990. GMP-140 mediates adhesion of stimulated platelets to neutrophils. Blood. 75:550-554.

34. Mayadas, T. N., R. C. Johnson, H. Rayburn, R. O. Hynes, and D. D. Wagner. 1993. Leukocyte rolling and extravasation are severely compromised in P selectin-deficient mice. Cell. 74:541-554.

35. Mulligan, M. S., M. J. Polley, R. J. Bayer, M. F. Nunn, J. C. Paulson, and P. A. Ward. 1992. Neutrophil-dependent acute lung injury. Requirement for P-selectin (GMP-140). J. Clin. Invest. 90:1600-1607.

36. Winn, R. K., D. Liggitt, N. B. Vedder, J. C. Paulson, and J. M. Harlan. 1993. Anti-P-selectin monoclonal antibody attenuates reperfusion injury to the rabbit ear. J. Clin. Invest. 92:2042-2047.

37. El Nahas, A. M. 1993. Masugi nephritis: a model for all seasons. In Experimental and Genetic Rat Models of Chronic Renal Failure. N. Gretz and M. Strauch, editors. Karger, Basel. 49-67.

38. Weksler, B. B. 1992. Platelets. In Inflammation: Basic Principles and Clinical Correlates. J. I. Gallin, I. M. Goldstein, and R. Snyderman, editors. Raven Press, New York. 727-746.

39. Johnson, R. J., J. Floege, W. G. Couser, and C. E. Alpers. 1993. Role of platelet-derived growth factor in glomerular disease. J. Am. Soc. Nephrol. 4:119128

40. Border, W. A., and N. A. Noble. 1993. From serum sickness to cytokinesadvances in understanding the molecular pathogenesis of kidney disease. Lab Invest. 68:125-128. 